\author{
UNIVERSIDADE DE SÃO PAULO \\ INSTITUTO DE FÍSICA DE SÃO CARLOS
}

NOEL ARAUJO MOREIRA

Localization versus subradiance in three-dimensional scattering of light

São Carlos 



\title{
Localization versus subradiance in three-dimensional scattering of light
}

\author{
Dissertation presented to the Graduate Pro- \\ gram in Physics at the Instituto de Física de \\ São Carlos, Universidade de São Paulo, to \\ obtain the degree of Master in Science. \\ Concentration area: Applied Physics \\ Option: Computational Physics \\ Supervisor: Prof. Dr. Romain Pierre Marcel \\ Bachelard
}

Corrected version

(Original version available on the Program Unit)

\section{São Carlos}


I AUTHORIZE THE REPRODUCTION AND DISSEMINATION OF TOTAL OR PARTIAL COPIES OF THIS DOCUMENT, BY CONVENCIONAL OR ELECTRONIC MEDIA FOR STUDY OR RESEARCH PURPOSE, SINCE IT IS REFERENCED.

Araujo Moreira, Noel

Localization versus subradiance in three-

dimensionalscattering of light / Noel Araujo Moreira;

advisor Romain Pierre Marcel Bachelard - revised version -

- São Carlos 2019.

$40 \mathrm{p}$.

Dissertation (Master's degree - Graduate Program in Física Aplicada Computacional) -- Instituto de Física de São Carlos, Universidade de São Paulo - Brasil, 2019.

1. Localization. 2. Light Scattering. 3. Dipole-dipole interaction. 4. Phase Transition. I. Bachelard, Romain Pierre Marcel, advisor. II. Title. 


\section{ACKNOWLEDGEMENTS}

This study was financed in part by the Coordenação de Aperfeiçoamento de Pessoal de Nível Superior - Brasil (CAPES) - Finance Code 001.

I wish to thanks many names for different reasons. To make my life simple, I will only list some of them by first name, and nickname when applicable: Ana, Alfredo, Bruno, Edmilson(Cris), Guilherme(Aluguel), Guilherme(Primão), Julia(Alquimista), Leonardo, Marcos, Natali, Nicolas, Nicole(Fu), Paulo, Pedro(Ash), Pedro(PH), Philippe, Robin, Thiago, Vitor.

In this journey, I must thank Romain Bachelard. Even with my effort to make everythink correct, the number of bugs/mistakes outperforms the number of results in orders of magnitude, even so, he had patience with me. 

"I see now that the circumstances of one's birth are irrelevant. It is what you do with the gift of life that determines who you are."

"Once you eliminate the impossible, whatever remains, no matter how improbable, must be the truth." 



\section{ABSTRACT}

\section{MOREIRA, N. Localization versus subradiance in three-dimensional}

scattering of light. 2019. 40p. Dissertation (Master in Science) - Instituto de Física de São Carlos, Universidade de São Paulo, São Carlos, 2019.

A blue sky, a white cloud or a red sunset are explained by classical multiple scattering theory of light. However, these phenomena neglect interference occurrence. Once it is taken into account, interference in a disordered medium may actually put a halt to the propagation of light, an effect known as Anderson Localization. Until now, experimental reports of Anderson Localization of light in 3D systems have not been conclusive. Our goal is to understand what are the underlying obstacles, and look for new insights from a theoretical point of view. In this dissertation, the properties of a cloud of two-level atoms scattering light are investigated. The dipole-dipole interaction generates collective modes, some of them, being localized. We found that finite-size effects dominate the lifetime of the localized modes, specifically by the ratio of localization length to their distance to the system boundaries. Localized modes saturates at maximum of $20 \%$ even above phase transition. Studying the steady-state regime, the coupling between localized modes and light is weak. Both results agrees with the difficulty of experimental evidence of light localization and promote the link of experiments and theory.

Keywords: Localization. Light Scattering. Dipole-dipole interaction. Phase Transition. 



\section{RESUMO}

\section{MOREIRA, N. Localização versus sub-radiância no espalhamento}

tridimensional de luz. 2019. 40p. Dissertação (Mestrado em Ciências) - Instituto de Física de São Carlos, Universidade de São Paulo, São Carlos, 2019.

Um céu azul, uma nuvem branca ou um por do sol vermelho são explicados pela teoria clássica de espalhamento múltiplo da luz. No entanto, esses fenômenos negligenciam a ocorrência de interferências. Uma vez levada em conta, a interferência em um meio desordenado pode interromper a propagação da luz, um efeito conhecido como Localização de Anderson. Até agora, relatos experimentais de Anderson Localização de luz em sistemas 3D não foram conclusivos. Nosso objetivo é entender quais são os obstáculos fundamentais, e buscar novos insights do ponto de vista teórico. Nesta dissertação, as propriedades de uma nuvem de átomos de dois níveis espalhando luz de é investigado. A interação dipolo-dipolo gera modos coletivos, alguns deles, sendo localizados. Descobrimos que os efeitos de tamanho finito dominam o tempo de vida dos modos localizados, especificamente pela razão entre o comprimento da localização e sua distância até os limites do sistema. Os modos localizados saturam no máximo 20\%, mesmo acima da transição de fase. Estudando o regime de estado estacionário, o acoplamento entre modos localizados e luz é fraco. Ambos os resultados concordam com a dificuldade da evidência experimental da localização da luz e promovem a ligação entre experimentos e teoria.

Palavras-chave: Localização. Espalhamento de luz. Interação dipolo-dipolo. Transição de fase. 



\section{LIST OF FIGURES}

Figure 1 - The intensity of the random potential is crucial to produce localized modes. . . . . . . . . . . . . . . . . . . . . . . 19

Figure 2 - Eigenspectrum $\left(\Im\left(\lambda_{n}\right), \Re\left(\lambda_{n}\right)\right.$ for a system with a density ten times larger than the critical one $\left(\rho=k^{3}, k l=0.12\right), N=6000$ and $\Delta=0$. Superradiant, subradiant and localized modes are indicated by triangles, circle and square markers, respectively. The insets show typical spatial profiles of each kind of mode. For the localized mode (a), the red markers denote the particles used to determine the localization length of the mode, and the line of corresponding fit.

Figure 3 - Relative proportion $P_{\omega}$ of localized and subradiant modes for a narrow $(\delta \omega=0.021)$ range of energy, as a function of the system density. Simulations realized with $N=1300$ particles. A threshold of 15 was imposed on the number of modes in that energy range to plot the resulting proportions. The proportion of superradiant modes is not represented, as their number is usually below the threshold. . . . . . . . . . . . . . . . 24

Figure 4 - Relation between the IPR and $R_{1}$ of the scattering modes. The localized/subradiant/superradiant modes are marked as squares/disks/triangles. Same parameters as Figure 2. . . . . . . . . . . . . . . . . . 25

Figure 5 - Proportion of localized, subradiant and superradiant modes for an increasing density and a fixed number of particles $(N=1500$ and 7000$)$.

Figure 6 - In the context of the Metal-Insulator transition, we identify phase transition by the collapse of Probability distribution $P(\tilde{\alpha}) \ldots \ldots . . .$.

Figure 7 - Differents $P(\tilde{\alpha})$ using the eigenvector associated with lowest $\Gamma_{n}$ at $\rho=$ $0.1 k^{2}$

Figure 8 - Population in the localized, subradiant and superradiant modes to a plane-wave, as a function of density and detuning. Simulations realized with a particle number $N=1500$, with an averaging over 100 realizations for each value of detuning and density. The gray area for low densities correspond to a regime of low resonant optical thickness, where superradiance and subradiance are not well formed; this area reduces as the number of particles is increased. . . . . . . . . . . . . .

Figure 9 - (Absence of) Correlations between the decay rate and the localization length of the localized modes. Simulation realized for a cloud of density $\rho=1(k l=0.12)$, with $N=9000$ particles. 
Figure 10 - Decay rate of the modes as a function of the ratio between their distance to the boundary $r^{\prime}=\left(R-\left|\mathbf{r}_{\mathbf{C M}}^{n}\right|\right)$ and their localization length $\xi$. The line corresponds to $\Gamma_{n}=C \exp \left(-r^{\prime} / \xi_{n}\right)$, with $C=2.10^{-3}$. The simulation for density $\rho=0.5 k^{3}(k l=0.2)$, resp. $50 k^{3}(k l=0,002)$, presents localization lengths of order $\xi \sim 0.07 / k$, resp. $\sim 0.4 / k$. Simulations realized with $N=4000$. . . . . . . . . . . . . . . 32

Figure 11 - Light decay for a system of $N=1500$ and averaged over 100 realizations. 34 


\section{LIST OF SYMBOLS}

$\begin{array}{ll}\beta & \text { Dipole Excitation } \\ \Gamma & \text { Inverse Decay Rate } \\ \lambda & \text { Eigenvalues } \\ \Psi & \text { Eigenvectors } \\ \xi & \text { Localization Length } \\ \Omega & \text { External Electric Field }\end{array}$





\section{CONTENTS}

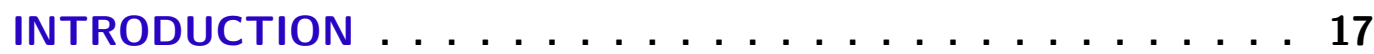

$1.1 \quad$ A brief introduction to Anderson Localization . . . . . . . . . . 18

2 COUPLED DIPOLE MODEL . . . . . . . . . . . . 21

2.1 Definitions . . . . . . . . . . . . . . . 21

$2.2 \quad$ Inverse Participation Ratio . . . . . . . . . . . . . . 23

$2.3 \quad$ Phase transition . . . . . . . . . . . . . . . . 25

3 LIGHT-MATTER INTERACTION . . . . . . . . . . . . . 29

3.1 Distribution of modes and their coupling to the exterior world . . 29

$3.2 \quad$ Intensity Decay . . . . . . . . . . . . . . . . . . . 32

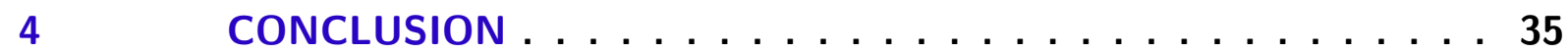

REFERENCES . . . . . . . . . . . . . . . . 37 



\section{INTRODUCTION}

Since the pioneering work by Philip W. Anderson ${ }^{1}$ to explain the transition from metal to insulator for electronic transport, the trapping of waves into disordered potential has been achieved in a variety of systems, ranging from acoustics to matter-waves. ${ }^{2-5}$ As for electromagnetic waves, localization has been reported in $1 \mathrm{D}^{6}$ and $2 \mathrm{D},{ }^{7}$ yet the $3 \mathrm{D}$ case has been the subject of an ongoing controversy. ${ }^{8}$ Indeed, the initial signatures used in the diffuse transmission or in the late time dynamics were later questioned, ${ }^{9-15}$ and at the moment, an unambiguous signature is lacking.

From a fundamental point of view, the vectorial nature of electromagnetic waves has been shown to prevent localization. ${ }^{16,17}$ This theoretical analysis was based on the scaling analysis, ${ }^{18}$ where the linewidth of the modes (i.e., their inverse lifetimes) is compared to the typical difference of frequency between them. The transition to localization is thus marked by the rise of modes with long lifetimes.

However, collective scattering modes can have diverse origins. A paradigmatic example was introduced with superradiance, where the scatterers undergo a series of symmetric states and radiate in an accelerated manner. ${ }^{19}$ Its counterpart, subradiance, is characterized by a radiation slower than the single-scatterer one, and it has been observed in atomic samples. ${ }^{20,21}$ Since both scattering modes can be observed in dilute regime, i.e., much below the critical density for localization, these long lifetimes are unrelated to localization.

The presence of subradiance in the dilute limit clearly questions the ability of latetime dynamics to capture the localization transition. ${ }^{13}$ But more generally, it challenges the idea of an all-localized scenario for the insulating phase of 3D optical systems. On the contrary, superradiance and subradiance may provide competing dynamical signatures to localization, and they do not require disorder. ${ }^{22}$ It is thus necessary to understand the interplay between these phenomena, in order to be, eventually, able to determine an unambiguous temporal signature of the localization transition.

We point out that despite the vectorial light waves may not localize, it was then shown that the usage of a strong magnetic field ${ }^{23}$ makes the scattering effectively scalar, and restores localization. Hence, we shall hereafter focus on a scalar model of 3D light scattering, which the localization transition has been well characterized. ${ }^{16,24-26}$

In this work, we study the competition between localized, subradiant and superradiant modes, showing that a transition to the localization regime is not achieved for the system sizes accessible numerically. Indeed, localized modes represent only a minority of modes, and despite their high densities in a specific range of energies, even an appro- 
priate choice of pump frequency does not prevent from strongly coupling to the other modes. Finally, the lifetime of these modes appear to be driven by a leakage due to the finite distance to the boundaries of the system. This allows us to evaluate the ratio of the localization length to their distance to the system's boundaries from the lifetime of the localized modes. These results contribute to the difficult task of finding unambiguous signatures of Anderson localization of light in 3D.

\subsection{A brief introduction to Anderson Localization}

This small section is devoted to illustrate Anderson Localization with a simple example. Using Anderson's original work as a starting point, ${ }^{1}$ one can consider a quantum free particle in a box size $L$. As wave function evolves, it scatters at spatial irregularities of the medium. If the medium is disordered enough, wave functions may interfere with themselves in such a way that they present a non-zero amplitude only at a specific region of space, that is, an exponential decay. For instance, let us study the wave functions are obtained by solving the Schrodinger's equation:

$$
\hat{H}|\Psi\rangle=\left(-\frac{1}{2} \nabla^{2}+U\right)|\Psi\rangle=E|\Psi\rangle .
$$

We will simulate a problem analogous to an infinite well, but changing the null potential into an uniform random distribution. For simplicity, we restrict to the onedimensional case, setting all constants equal to unity.

To solve this equation numerically, one must formulate $H$ in such a way that the computer can calculates its eigenvalues and eigenvectors. Garcia, Zozulya e Stickney ${ }^{27}$ provides a detailed explanation, but in essence the continuous domain $L$ is divided in discrete intervals of size $\delta x$. The wave function at position $j$ get the notation $|\Psi\rangle_{j}$. For the Laplacian operator, $\mathrm{Kutz}^{28}$ presents the following matrix:

$$
\nabla^{2}=\frac{1}{\delta x^{2}}\left(\begin{array}{cccccc}
-2 & 1 & 0 & 0 & \ldots & 0 \\
1 & -2 & 1 & 0 & \ldots & \\
0 & 1 & -2 & 1 & \ldots & \\
& & \vdots & & & \\
& \ldots & & 1 & -2 & 1 \\
0 & \ldots & & 0 & 1 & -2
\end{array}\right)
$$

To create the matrix potential $U$, we simply generate random numbers using the standard rand() function of our favorite programming language.

Figure 1 presents two random potential and the square modulus of the associated wave functions. For small values of $U$ (Figure 1a) results are essentially identical to the free particle problem and the distribution is considered extended, because each wave function 
(a)

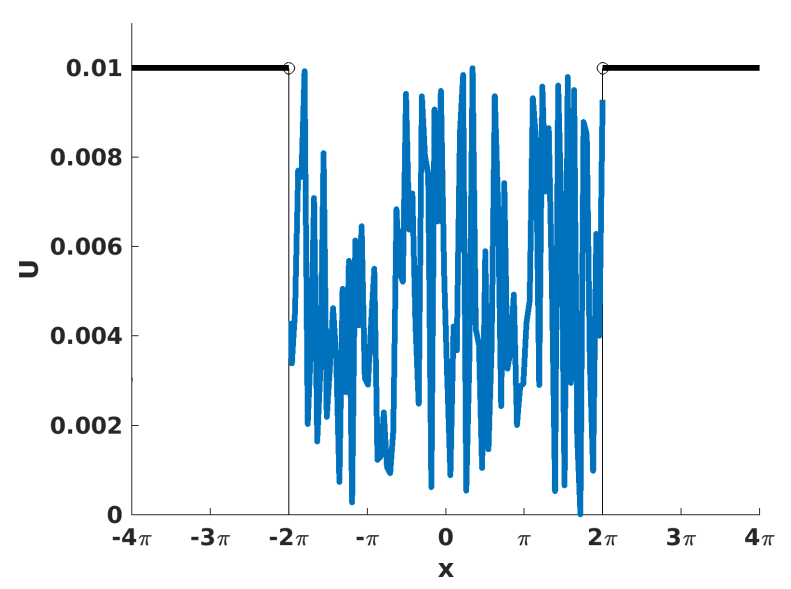

(c)

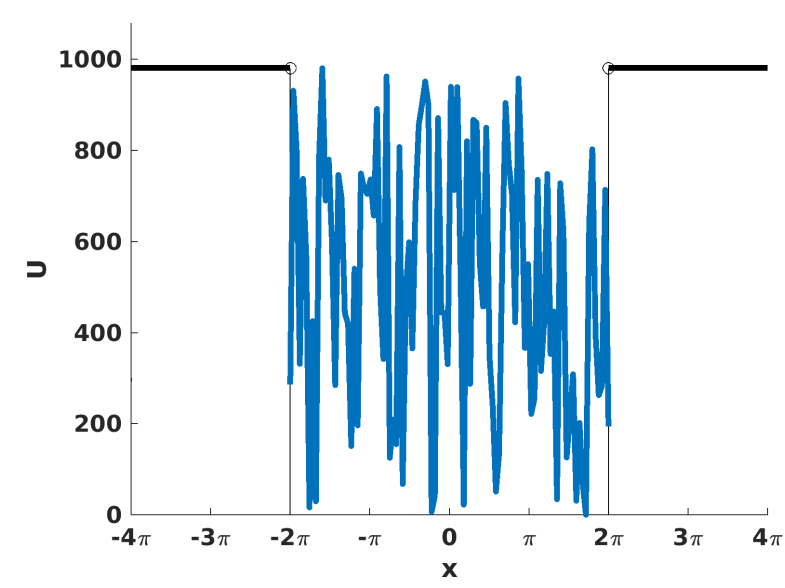

(b)

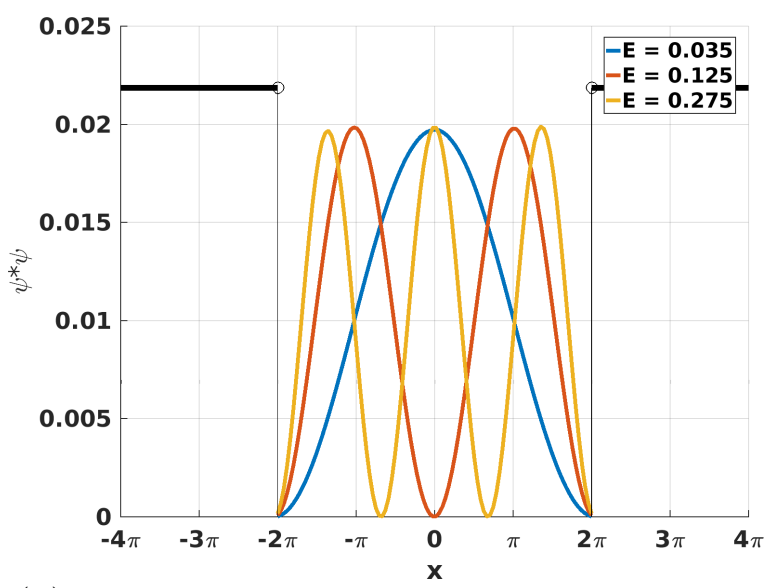

(d)

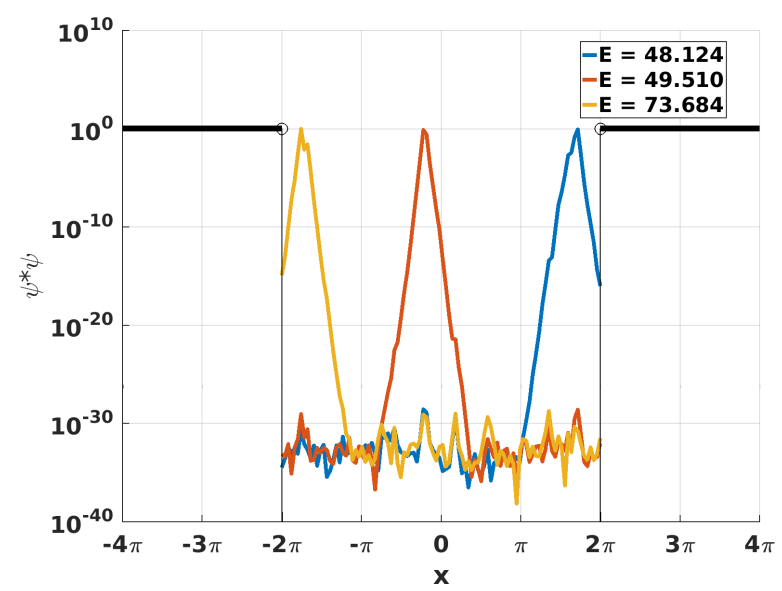

Figure 1 - The intensity of the random potential is crucial to produce localized modes. Source: By the author.

extend over the entire system. Increasing the intensity of the potential, we observe that the squared modulus of the wave functions are concentrated in a certain region, that is, they are localized as in Figure 1d. 



\section{COUPLED DIPOLE MODEL}

\subsection{Definitions}

We consider a model of $N$ point-like scatterers with positions $\mathbf{r}_{j}$ distributed randomly and homogeneously inside a spherical cloud of radius $R$. This cloud has $\rho=N / V$, and optical thickness, $b_{0}=4 N / k^{2} R^{2}$ ( $k$ the wavenumber of the scattered light), so we have: $R=\frac{3 b_{0}}{16 \pi \rho}$ and $N=\frac{4 \pi}{3} \rho R^{3}$. The scatterers can be thought as two-level atoms with linewidth $\Gamma$ and transition frequency $\omega_{a}$. The system is shined with a monochromatic wave Rabi frequency $\Omega(\mathbf{r})$ and frequency $\omega=k c$, detuned by $\Delta=\omega-\omega_{a}$ from the atomic transition is given in units of $\Gamma$. The exchange of virtual and real photons between the particles result in an effective interaction between the atomic dipoles $\beta_{j}$. In the linear optics regime, the dipole dynamics is governed by a set of coupled differential equations ${ }^{29}$

$$
\frac{d \beta_{j}}{d t}=\left(i \Delta-\frac{\Gamma}{2}\right) \beta_{j}-\frac{\Gamma}{2} \sum_{m \neq j} \frac{\exp \left(i k\left|\mathbf{r}_{j}-\mathbf{r}_{m}\right|\right)}{i k\left|\mathbf{r}_{j}-\mathbf{r}_{m}\right|} \beta_{j}-\frac{i \Omega(\vec{r})}{2}
$$

Remark that the model can also be derived from fully classical principles. ${ }^{22,30}$ The first right-hand term describes the single-dipole dynamics, the second one corresponds to the dipole-dipole coupling, and the last one to the pump. In order to avoid contributions from pair physics, ${ }^{31}$ we set a exclusion volume in the particle distribution, based upon the density: $r_{\min }=\rho^{-1 / 3} / \pi$. Throughout this work, the pump considered is a plane-wave travelling in the $\hat{z}$ direction: $\Omega\left(\vec{r}_{j}\right)=\Omega e^{i \mathbf{k}_{0} \cdot \mathbf{r}_{j}}$, with $\mathbf{k}_{0}=k \hat{z}$; we have checked that using a Gaussian beam with a waist comparable to the system size does not alter our conclusions.

A bare estimate of mean free path of the sample is $k l=1 / \sigma \rho . \sigma$ is the atom scattering cross section.

The dipole-dipole coupling results in collective scattering modes, with an associated decay rate and frequency. That is, the atomic cloud as a whole presents a new set of decay rates and frequencies. To see that, is convenient to write equation 2.1 in matrix form:

$$
\frac{d \vec{\beta}}{d t}=\mathbf{M} \vec{\beta}-i \frac{\vec{\Omega}}{2},
$$

with $M_{j m}=\delta_{j m}(i \Delta-\Gamma / 2)+\left(1-\delta_{j m}\right) \exp \left(i k\left|\mathbf{r}_{j}-\mathbf{r}_{m}\right|\right) /\left(i k\left|\mathbf{r}_{j}-\mathbf{r}_{m}\right|\right)$, where $\delta_{j m}$ referring to the Kronecker symbol. The eigenvalues of $M, \lambda_{n}$ decompose as the decay rates $\Gamma_{n}=$ $-\Re\left(\lambda_{n}\right)$ and frequency shifts $\omega_{n}=\Im\left(\lambda_{n}\right)$ from the atomic transition. The components $\left|\Psi_{j}^{n}\right|^{2}$ represents the excitation contribution of atom $j$ to mode $n$.

In this system, the strength of the disorder is controlled through the atomic density. Superradiant and subradiant modes emerges from the dipole-dipole coupling, as well as 


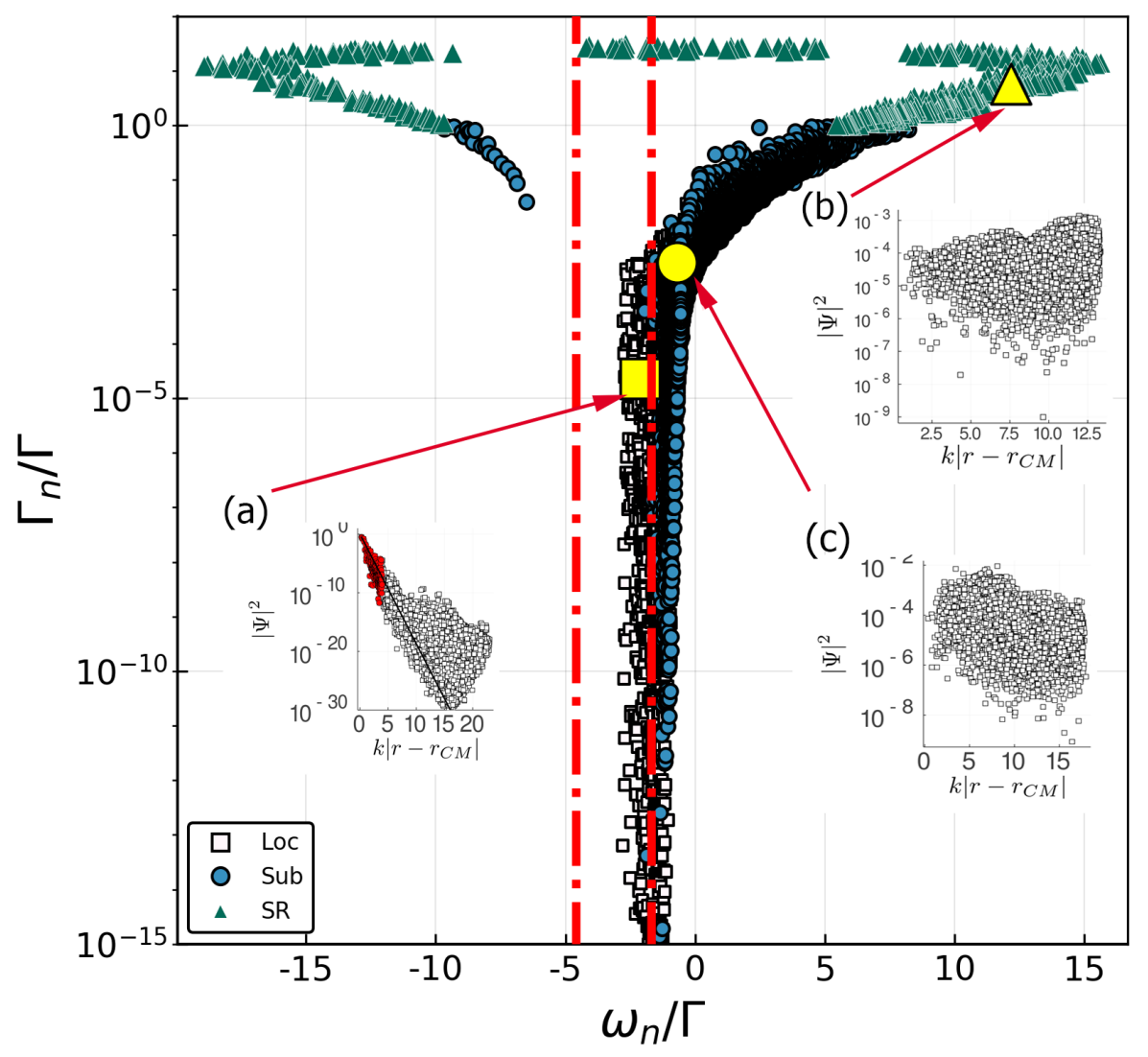

Figure 2 - Eigenspectrum $\left(\Im\left(\lambda_{n}\right), \Re\left(\lambda_{n}\right)\right.$ for a system with a density ten times larger than the critical one $\left(\rho=k^{3}, k l=0.12\right), N=6000$ and $\Delta=0$. Superradiant, subradiant and localized modes are indicated by triangles, circle and square markers, respectively. The insets show typical spatial profiles of each kind of mode. For the localized mode (a), the red markers denote the particles used to determine the localization length of the mode, and the line of corresponding fit.

Source: By the author.

exponentially localized modes above the transition threshold $\rho_{c} \approx 0.1 k^{3}(k l \approx 1.1),{ }^{16,24}$ with long lifetimes. Consequently, we decompose modes into three categories, which are illustrated in Figure 2, along the eigenspectrum: (a) Localized modes, that present an exponentially decaying spatial profile [see Fig.2(a)]; (b) Superradiant modes, that present a decay rate $\Gamma_{n}>1$ [see Fig.2(b)]; (c) Subradiant modes $\left(\Gamma_{n}<1\right.$ ) that do not present an exponentially decaying spatial profile [see Fig.2(c)].

The spatial profiles presented in the insets of Figure 2 describe the particles contribution, ordered from their distance to the mode center-of-mass $\mathbf{r}_{\mathrm{CM}}^{n}=\sum_{m}\left|\Psi_{m}^{n}\right|^{2} \mathbf{r}_{m}$. They are typical profiles of the three kinds of modes encountered in the system. Superradiant and subradiant are rather extended, whereas localized modes present, for short distances, an exponential decay. Indeed, in the open system (2.1), localized modes present a spatial profile which decays exponentially at first, before presenting a much slower decay. ${ }^{32,33}$ The determination of the localization length $\xi$ that characterizes the decay 
$\left(|\Psi(\mathbf{r})|^{2} \sim \exp \left(\left|\mathbf{r}-\mathbf{r}_{\mathrm{CM}}\right| / \xi\right)\right)$ of these hybrid modes* must thus be realized by giving a larger weight to the atoms contributing most to the mode. This is achieved using the $L_{1}$ minimization fitting procedure to compute $\xi$ from the $|\Psi(\mathbf{r})|^{2}$ profile, without logarithmic rescaling, considering only the atoms closest to the mode center-of-mass and that altogether represent $99.995 \%$ of the mode norm. The minimization of the $L_{1}$ aims to minimize the absolute difference of errors (quantified by the Adapted Coefficient of Determination $R^{1}$ ), rather than the usual square error (i.e., the $R^{2}$ ), and has been used to overcome outliers issues. ${ }^{28,34,35}$ Throughout this work, we require for a mode to present a $R^{1}$ larger than 0.6 to be considered localized: An inspection of the profiles of various modes suggests that this value is a reasonable choice to distinguish the modes.

Figure 2 suggests that, even at a density ten times larger than the critical density for localization, a significant number of modes do not present an exponentially decaying profile: the scattering modes present a broad range of energies, the localized modes being concentrated over narrow band. Interestingly, the Ioffe-Regel criterion appears to predict correctly the edge between the localized and subradiant modes, once the Lorentz-Lorenz shift is included: ${ }^{36-38}$

$$
\frac{\Delta_{c}}{\Gamma}=-\frac{\pi \rho}{k^{3}} \pm \frac{1}{2} \sqrt{3 \pi \frac{\rho}{k^{3}}-1} .
$$

A refined analysis on the distribution of energy of these modes reveals that localized modes are concentrated over specific ranges of energy, bounded by the mobility edges. ${ }^{25}$ As can be observed in Fig.3, almost all modes are localized in the proper range of negative detuning, whereas in a different range of energy around the resonance, almost all modes are subradiant. Superradiant modes represent always a minority of modes, yet as we shall now see, their relative contribution cannot be neglected.

\subsection{Inverse Participation Ratio}

Superradiant and subradiant modes present a broad spatial profile whereas localized modes are characterized by the contribution of very few atoms (see Figure 2(a) and (b)). This is confirmed by the analysis of the Inverse Participation Ratio (IPR), defined as

$$
\mathrm{IPR}_{n}=\frac{\sum_{m}\left|\Psi_{m}^{n}\right|^{4}}{\left(\sum_{m}\left|\Psi_{m}^{n}\right|^{2}\right)^{2}}
$$

The value of $R^{1}$ cannot be directly associated to usual localization parameters such as the Ioffe-Regel criterion, since $R^{1}$ relates only to the mode profile, whereas the, Ioffe-Regel criterion relates to mean free path. It is thus more relevant to compare the $R^{1}$ to the IPR, that also relates to the mode profile.

* Even with exponential decay profile, some mode have tails that may not supress transmission, and the mode can be sensitivity to changes in the boundary conditions of the system. 


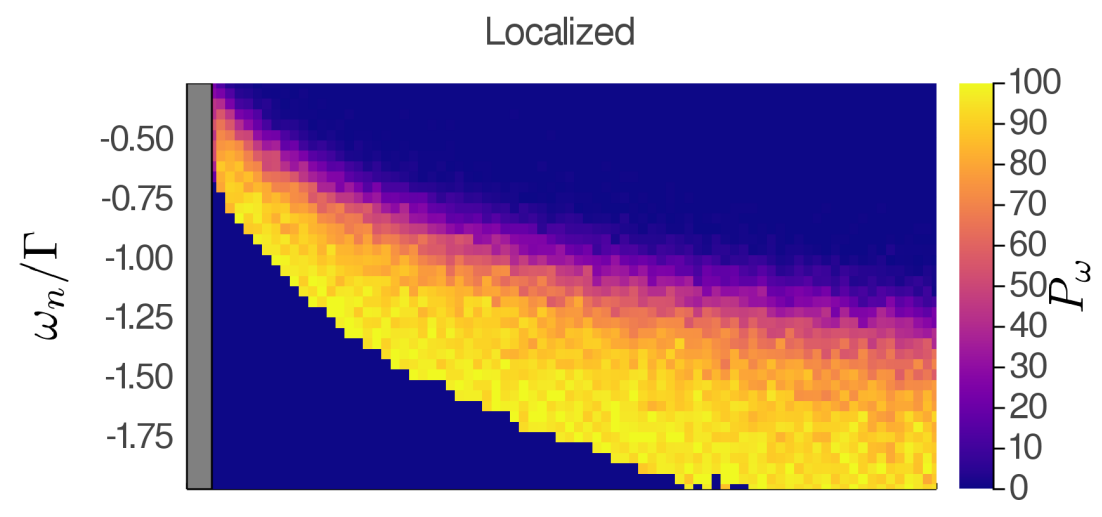

Subradiant

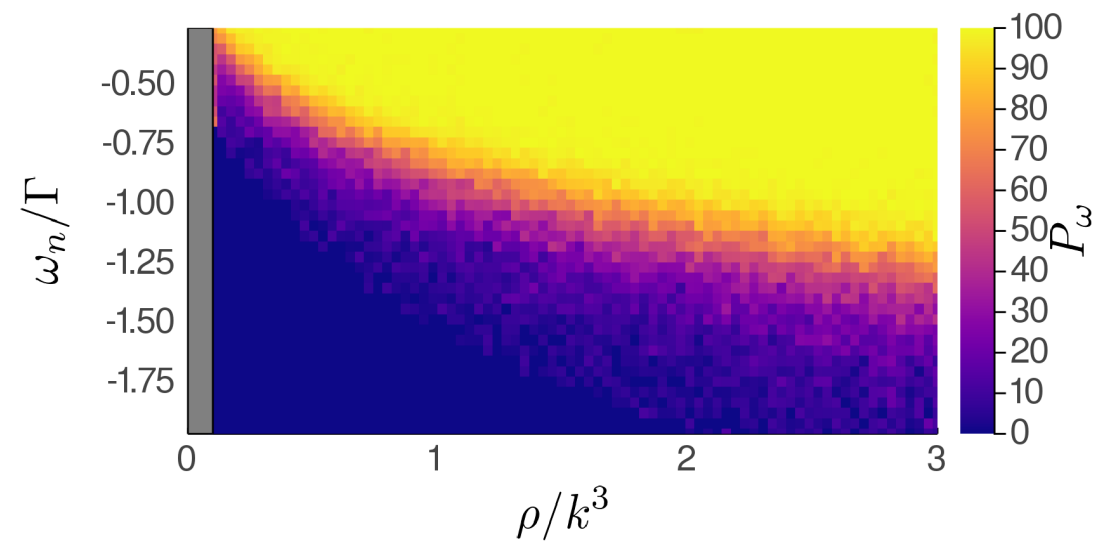

Figure 3 - Relative proportion $P_{\omega}$ of localized and subradiant modes for a narrow $(\delta \omega=0.021)$ range of energy, as a function of the system density. Simulations realized with $N=1300$ particles. A threshold of 15 was imposed on the number of modes in that energy range to plot the resulting proportions. The proportion of superradiant modes is not represented, as their number is usually below the threshold.

As can be observed in Figure 4, superradiant modes present an IPR of $\sim 10^{-3}$ (the simulations were realized for $N=5.10^{3}$, which naturally bounds the IPR to a lower value of $\left.1 / N=1 /\left(5.10^{3}\right)\right)$, while for localized modes present it is typically $0.2-0.5$. Subradiant modes exhibit a much broader variety of IPR, from $\sim 0.4$ to $10^{-3}$, that witness their extended nature. Overall, the strong correlation observed between the IPR and the $R^{1}$ fitting coefficient validates the use of the $R^{1}$ factor to classify the modes, considering that the IPR has been used extensively to study the Anderson localization transition. ${ }^{39}$

Note that we do not use information on the transmission of light to infer a classification of the modes. Therefore we acknowledge that additional physics may be explored, for example necklace modes, i.e., coupled resonances inside the sample that leads to peaks in transmission. ${ }^{40,41}$ 


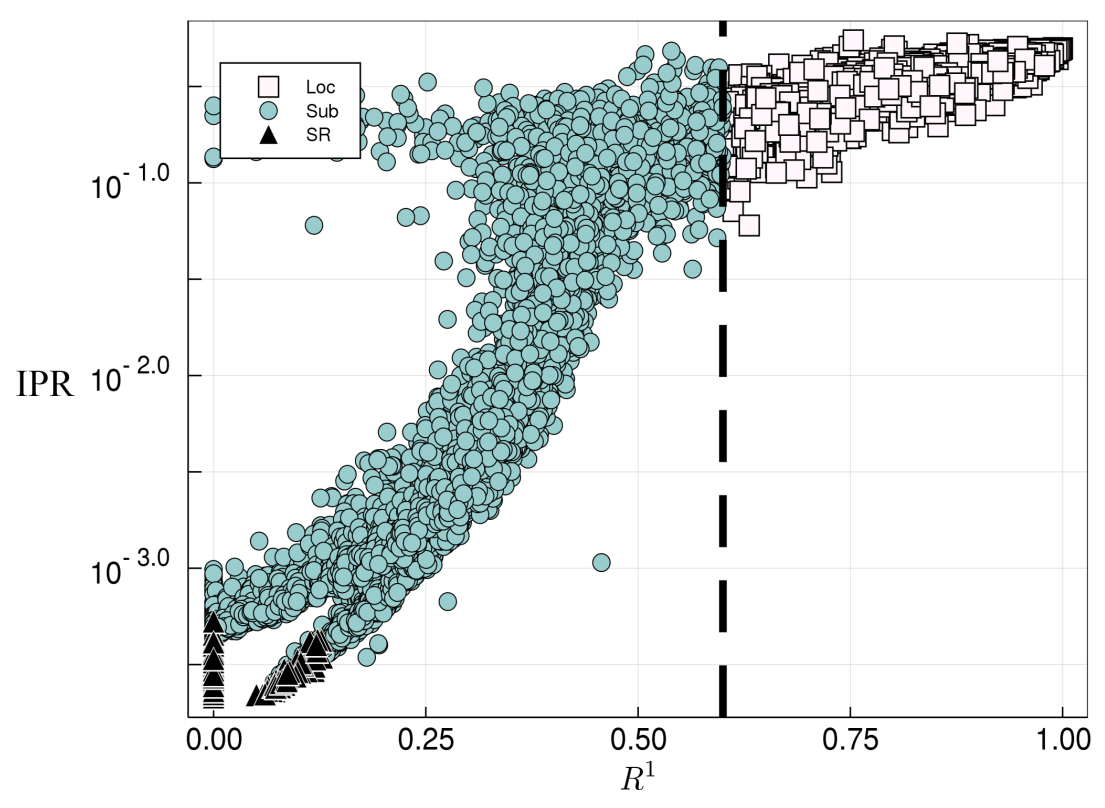

Figure 4 - Relation between the IPR and $R_{1}$ of the scattering modes. The localized/ subradiant/superradiant modes are marked as squares/disks/triangles.

Same parameters as Figure 2.

Source: By the author.

\subsection{Phase transition}

Figure 2 suggests that, even at ten times the critical density, a significant number of modes do not present an exponentially decaying profile. A more quantitative analysis is presented in Figure 5, where the proportion of each kind of modes is shown, for different densities and for a fixed particle number. We first note that the proportion of superradiant modes decreases with the increasing density. It can be understood from the fact that smaller volumes are associated to larger superradiant rates, and thus fewer of them (the trace of matrix $\mathbf{M}$ is $N(i \Delta-\Gamma / 2)$, independently of the cloud geometry); in the subwavelength regime, a single superradiant value has a rate that tends to $N$, all other ones being subradiant.

More surprisingly, the remaining modes are largely dominated by subradiant ones, which typically constitute $\sim 75 \%$ of $N$ modes. Indeed, even for a density ten times larger than the transition one, ${ }^{16}$ localized modes represent at most one-fifth of all modes. The scenario where all modes would localize, inducing a transition to an insulator, is clearly not valid here. Furthermore, working at fixed particle number implies that the system size changes significantly, which discards boundary effects to explain the predominance of subradiant modes over localized ones. Increasing the particle number from $N=1500$ to $N=7000$ leads to a slight decrease of the proportion of superradiant modes (as the optical thickness increases), the proportion of subradiant modes increasing correspondingly, but that of localized modes being almost unaltered. This clearly questions the notion of the 


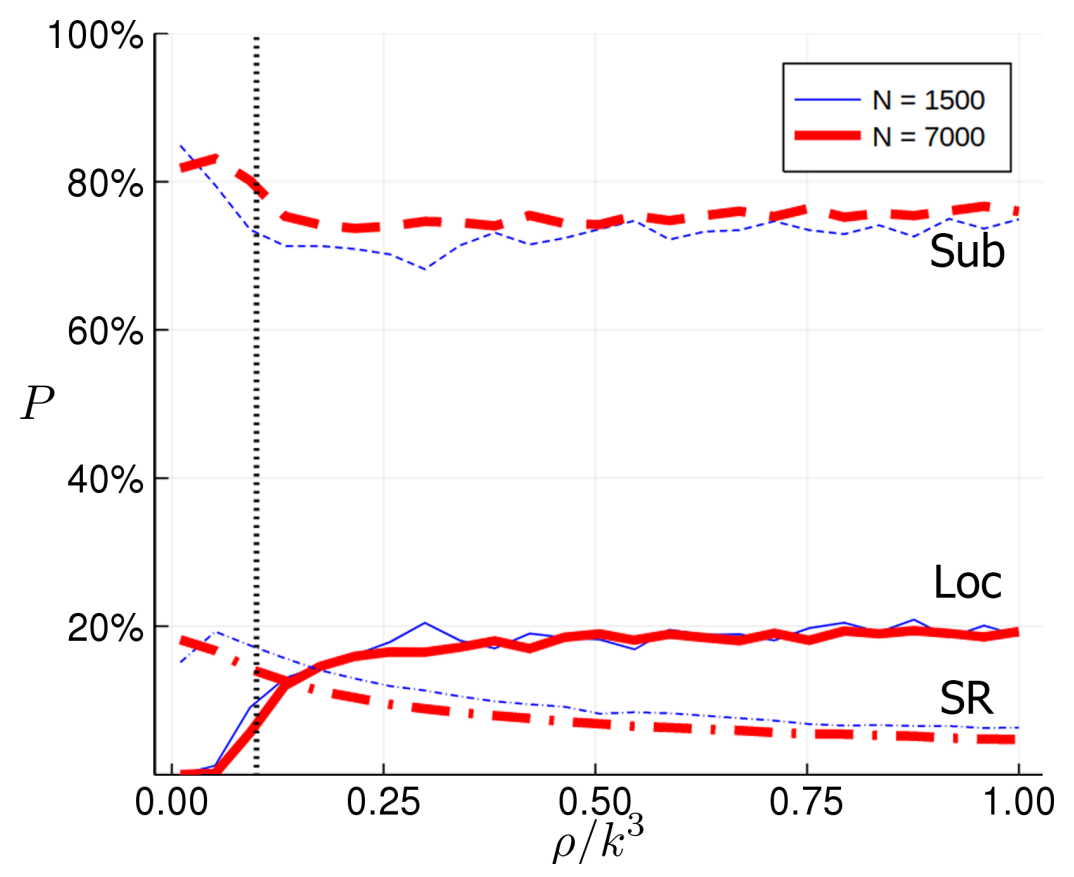

Figure 5 - Proportion of localized, subradiant and superradiant modes for an increasing density and a fixed number of particles $(N=1500$ and 7000$)$.

Source: By the author.

localization transition in that system, especially considering the existing debate on the possible signatures of the transition. For example, no exponential decay of the transmission with the system size, a paradigmatic signature of the localization regime, has been reported up to date. The existence of a large population of other modes (superradiant and subradiant), that could support the transport of light through the sample, may contribute to the lack of clear signatures of transition, despite a statistical eigenvalue analysis that favors long lifetimes modes predicts a transition. ${ }^{16}$

The study of phase transition in Anderson Localization is extensive and Evers e Mirlin ${ }^{42}$ characterized its multifractal nature using the Anderson Model. Here we just point out that we attempted (unsuccessfully) to obtain the critical exponents associated with phase transition using Multifractal Finite-Size Scaling. In general terms, any normalized probability distribution has Fractal Statistics if it has power law relation with system size - note that this power law usually is not associated with a mathematical fractal. Multifractal means that different regions of a domain have different power laws. ${ }^{43}$ Critical exponent associated to phase transition are defined on a infinite medium, finitesize scaling is a technique to handle the numerical constraint of system size. Multifractal finite-size scaling combines both concepts and aims to compute critical exponents without any relation to symmetry breaking.

The basic test to confirm phase transition is the collapse of the probability distribution $P(\tilde{\alpha})$ for different systems sizes, where $\tilde{\alpha}=-\ln |\Psi|^{2} / \ln R$. At the critical density, 


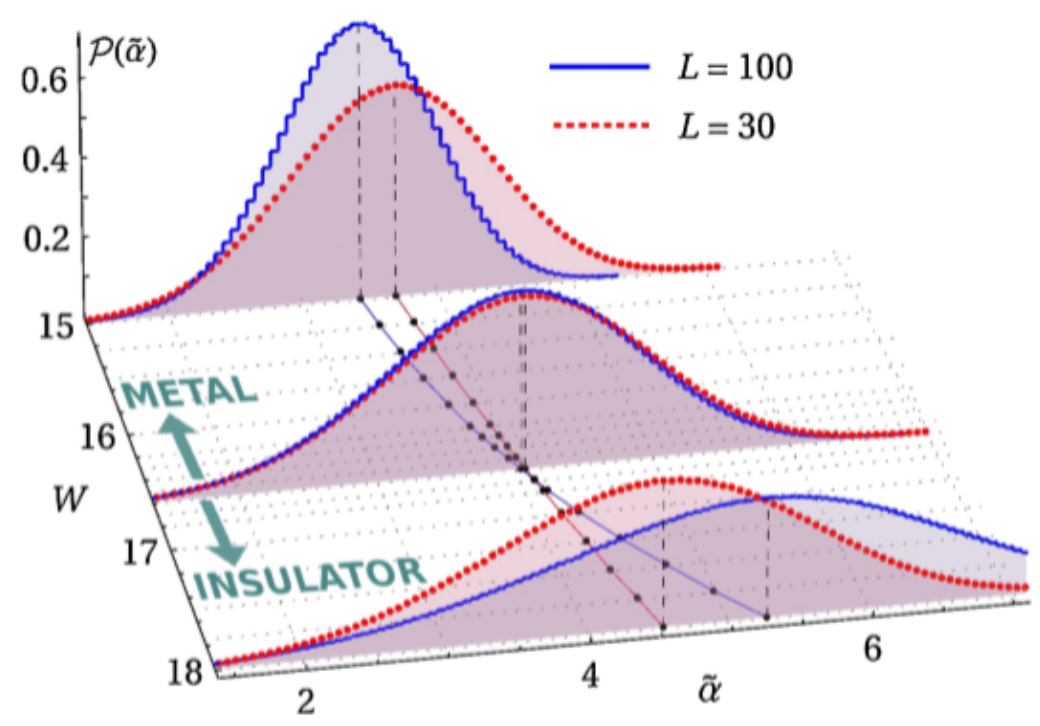

Figure 6 - In the context of the Metal-Insulator transition, we identify phase transition by the collapse of Probability distribution $P(\tilde{\alpha})$.

Source: RODRIGUEZ et al. ${ }^{44}$

for all system sizes, all $P(\alpha)$ curves collapse as shown in Figure 6. We were able to recreate the results of Figure 6 following the definitions of Ref 44, for the Anderson Model.

However, we did not have similar results when using the probability distribution data for the coupled dipole model. Our $P(\tilde{\alpha})$ presented too many oscillations and an average of large number of repetitions did not provide sign of convergence, Figure 7 is one sample result. One possible explanation is that the system size is too small for the thermodynamics assumption to be valid (the maximum number of particle simulated was $N=21000)$. The authors of Ref 44 indeed reach larger systems sizes since the Anderson Model has nearest neighbor interactions, which easily translates into sparse matrices, whereas the Coupled Dipole Model has long range interactions and required dense matrices. A second explanation is that the open nature of the Coupled Dipole Model may affect the validity of this approach. Finally, a third explanation is that our estimative for the critical density is inaccurate, and one needs to evaluate it more precisely before applying the Multifractal methology. Rather than investigating in more details these topics, we focused on the coupling of the modes to an external wave, which is discussed in the next section. 


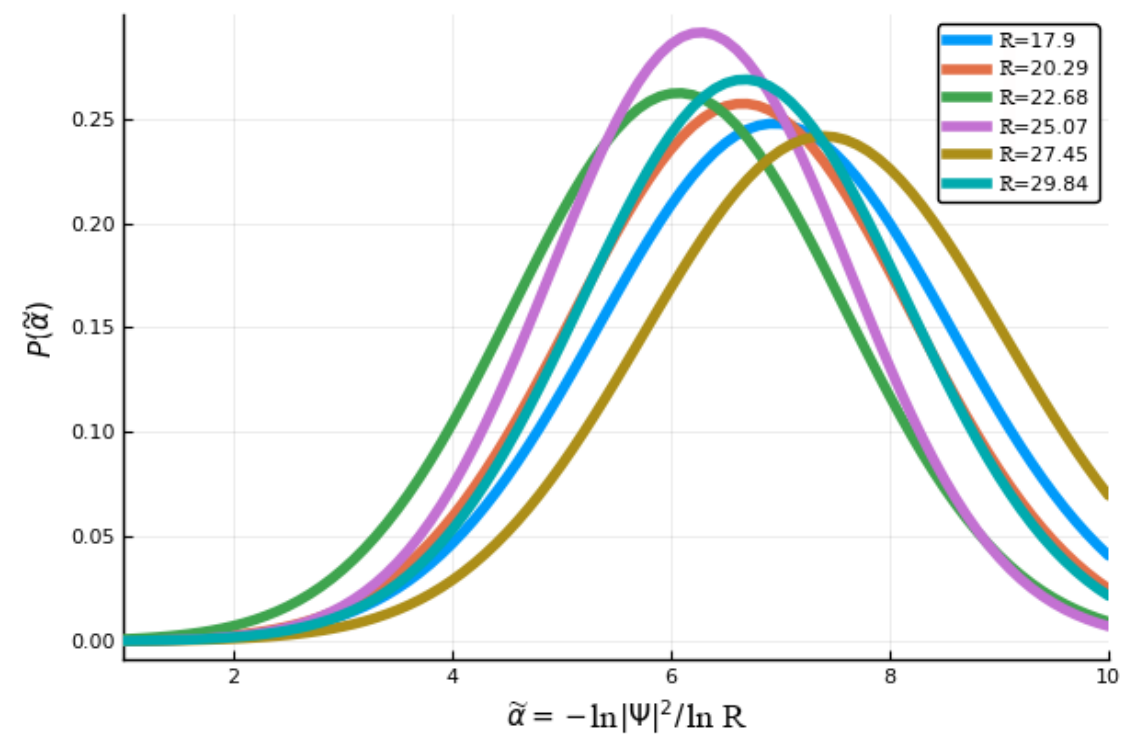

Figure 7 - Differents $P(\tilde{\alpha})$ using the eigenvector associated with lowest $\Gamma_{n}$ at $\rho=0.1 k^{2}$. Source: By the author. 


\section{LIGHT-MATTER INTERACTION}

\subsection{Distribution of modes and their coupling to the exterior world}

Localized modes represent a minority of all modes, yet an open question is how an incoming wave will couple to them, considering they present to a relatively narrow range of energies (see Figure 2). This coupling can be evaluated by studying the projection of the pump onto the scattering modes. The solution to equation 2.2 decomposes as:

$$
\vec{\beta}(t)=\sum_{n} \alpha_{n} \Psi_{n} e^{\lambda_{n} t}
$$

where $\alpha_{n}$ represents the projection coefficient. Thus, the set of $\left|\alpha_{n}\right|^{2}$ represents the population of the modes. ${ }^{45}$ We focus on the steady-state regime, for which the $\alpha_{n}$ s are obtained from equation 2.2 by:

$$
\vec{\alpha}=\frac{i}{2} \Psi^{-1} M^{-1} \vec{\Omega}
$$

From a numerical perspective, equation 3.2 is time consuming, specially to study its dependence on the different parameters. To achieve better performances, we make use of the fact that, when changing the detuning $\Delta$, its eigenvalues are only shifted by $i \Delta$, the eigenmodes being unaltered. This fact is useful because we can compute $\vec{\alpha}$ for a specific density and detuning. Then, we change $\Delta$ and apply equation 3.2 again without a new diagonalization of $M$.

In order to understand if it is possible to address the localized modes specifically, we study the mode population defined as

$$
P_{\text {Loc }}=\frac{\sum_{n \in\{\text { Loc }\}}\left|\alpha_{n}\right|^{2}}{\sum_{n=1}^{N}\left|\alpha_{n}\right|^{2}},
$$

where $\{$ Loc $\}$ refers to the ensembles of localized modes $\left(P_{\mathrm{SR}}\right.$ and $P_{\mathrm{Sub}}$ are defined in a similar way).

Because the localized modes belong to a narrow range of energies, the detuning $\Delta$ is tuned, in addition to the atomic density. As can be observed in Figure 8(a), the localized modes are addressed more specifically for a detuning of $\Delta \sim \Gamma$, yet even then, and for densities much beyond the critical one, at most half of the incoming plane-wave couples to it. The fact that at least half of the incoming wave projects on superradiant and subradiant modes may explain why a clear signature of the phase transition is difficult to identify. ${ }^{8}$ Indeed, these modes are unrelated to localization, as they are already present in the dilute regime, way below the critical density. ${ }^{21,46,47}$ 

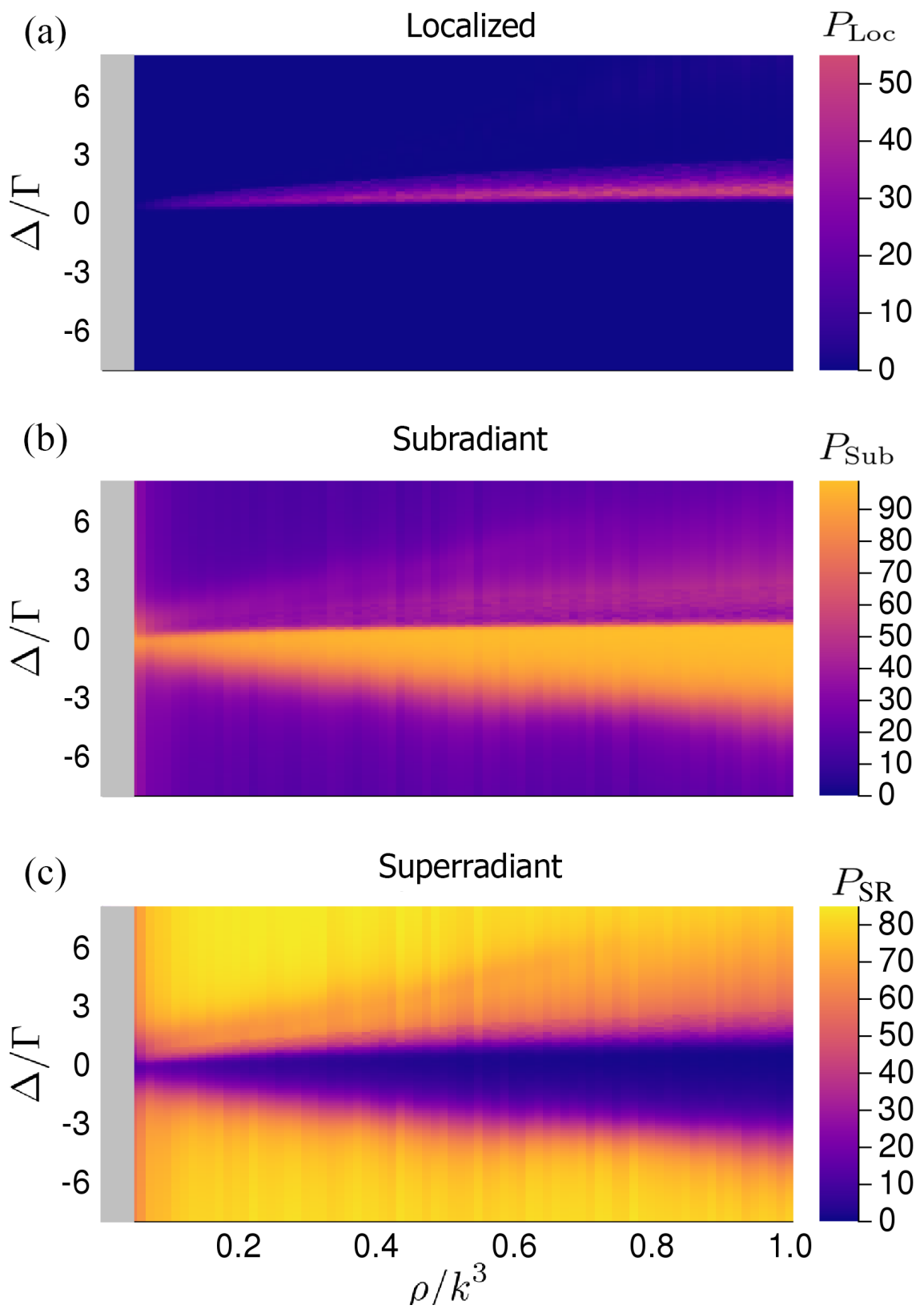

Figure 8 - Population in the localized, subradiant and superradiant modes to a plane-wave, as a function of density and detuning. Simulations realized with a particle number $N=1500$, with an averaging over 100 realizations for each value of detuning and density. The gray area for low densities correspond to a regime of low resonant optical thickness, where superradiance and subradiance are not well formed; this area reduces as the number of particles is increased.

Source: By the author. 


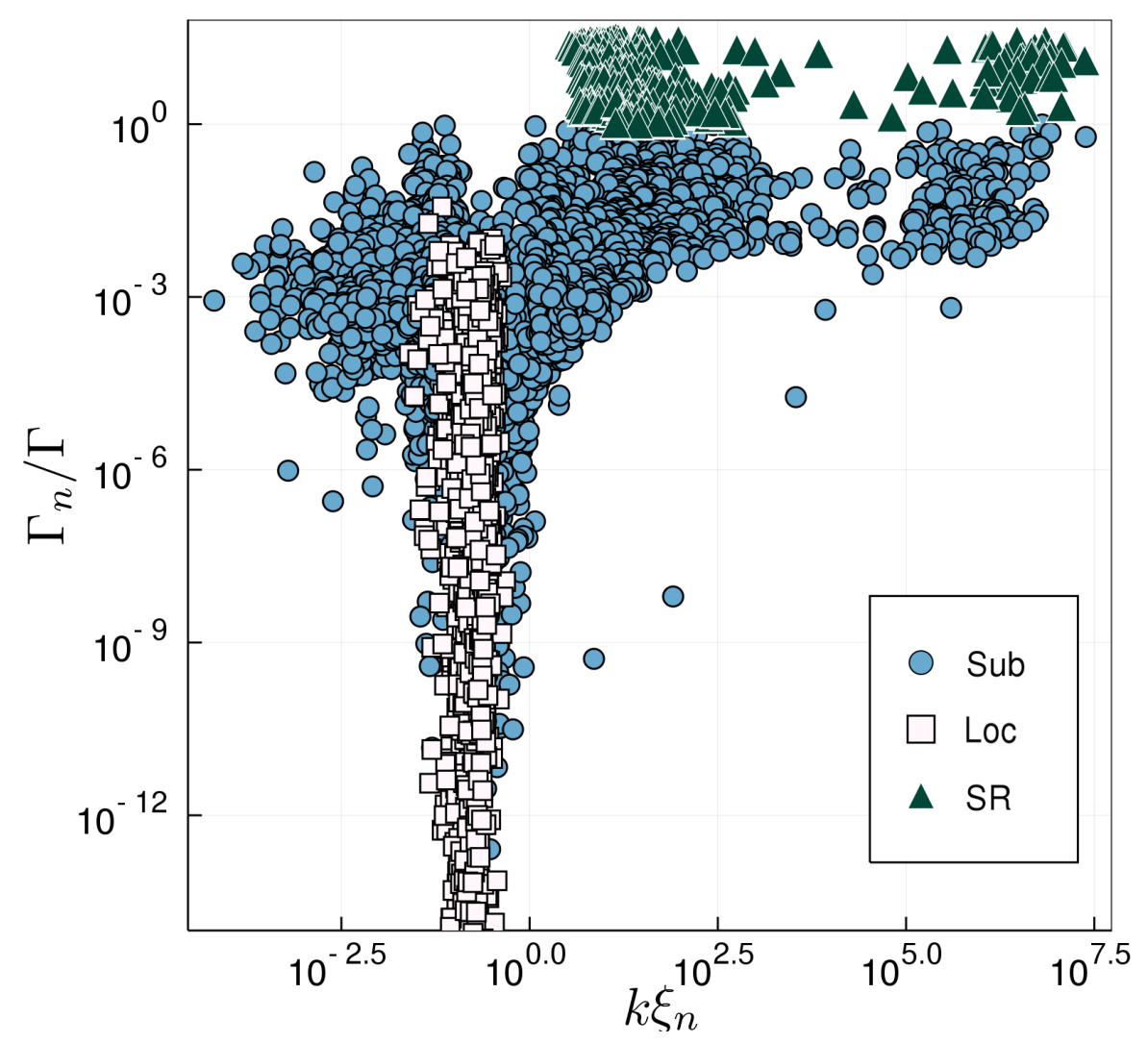

Figure 9 - (Absence of) Correlations between the decay rate and the localization length of the localized modes. Simulation realized for a cloud of density $\rho=1(k l=0.12)$, with $N=9000$ particles.

Source: By the author.

Subradiant modes are rather addressed using a negative, yet moderate, detuning (see Figure $8(\mathrm{~b})$ ), where almost all the plane wave may project on these. At large absolute values of the detuning, when the cloud turns transparent, superradiant modes are addressed predominantly. This is consistent with the picture of atoms illuminated homogeneously, with a phase given by that of the laser, as exemplified by the superradiant timed Dicke state. ${ }^{22,48}$ Note that we have checked that Gaussian beams with a waist smaller than the cloud radius (for a ratio up to one third) that the population of localized modes is not significantly altered.

The localized modes cover a broad range of lifetimes, that extends over many orders of magnitude, see Figure 9. Nevertheless, as it has been observed in the 2D case, ${ }^{17}$ there is no clear correlation between the lifetime and localization length of the modes.

The main source of the broadening in the modes linewidths can be found in finitesize effects: The proximity to the system boundary results in a leakage of the mode, which appears to be well described by $\Gamma_{\text {leak }}=C \exp \left(-r^{\prime} / \xi\right)$, ${ }^{49}$ with $r^{\prime}=R-\left|\mathbf{r}_{\mathbf{C M}}\right|$ the distance of the mode center-of-mass to the boundary and $C \approx 2 \cdot 10^{-3}$. This effect is illustrated in Figure 10, where a clear correlation between the system border and the mode lifetime is 


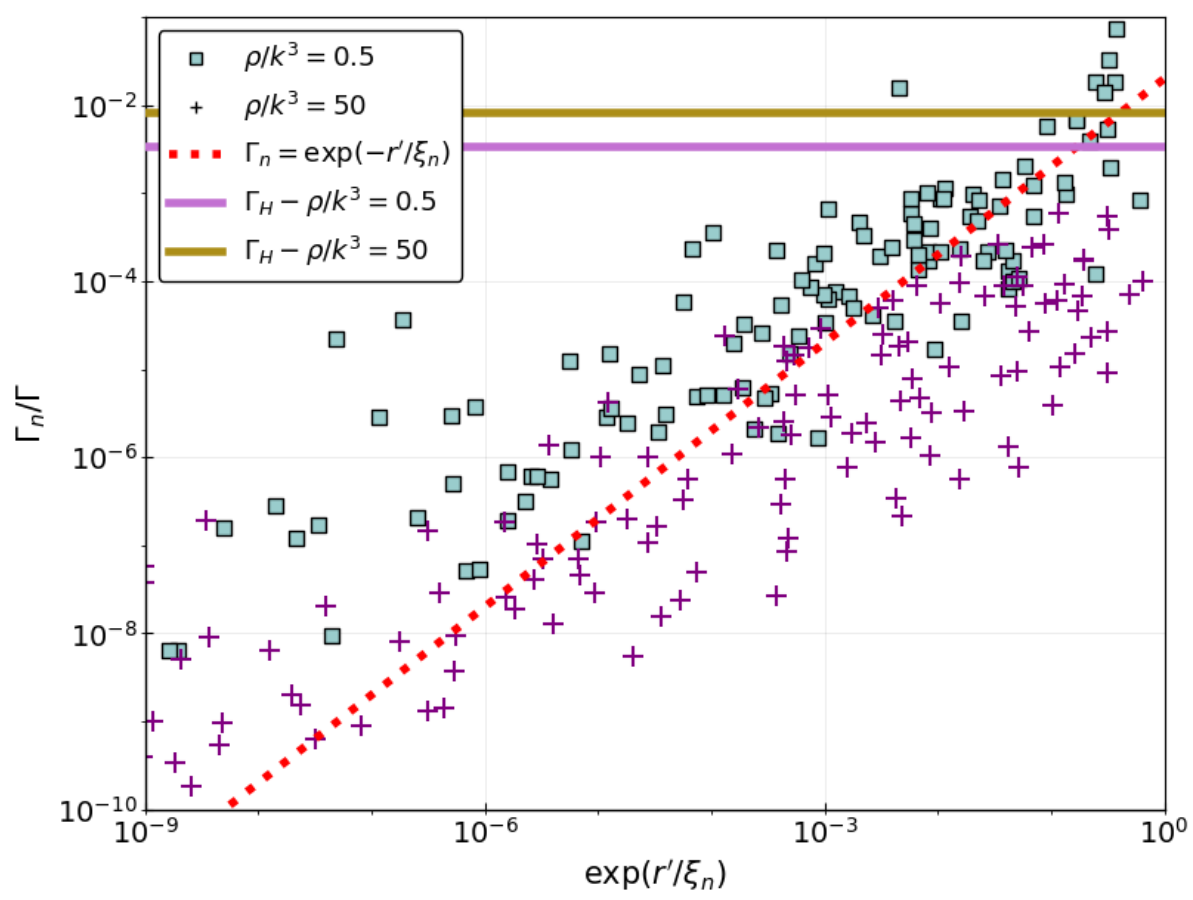

Figure 10 - Decay rate of the modes as a function of the ratio between their distance to the boundary $r^{\prime}=\left(R-\left|\mathbf{r}_{\mathbf{C M}}^{n}\right|\right)$ and their localization length $\xi$. The line corresponds to $\Gamma_{n}=C \exp \left(-r^{\prime} / \xi_{n}\right)$, with $C=2.10^{-3}$. The simulation for density $\rho=0.5 k^{3}(k l=0.2)$, resp. $50 k^{3}(k l=0.002)$, presents localization lengths of order $\xi \sim 0.07 / k$, resp. $\sim 0.4 / k$. Simulations realized with $N=4000$.

Source: By the author.

found, for two very different sets of parameters (corresponding to $\xi \sim 0.4 / k$ and $\sim 0.07 / k$ ). In the regime we have explored, the decay rates $\Gamma_{n}$ appear to be dominated by this effect, and the Heisenberg $\left(\Gamma_{H}\right.$ on Figure 10) and Thouless times, that describes respectively the energy separation between neighboring modes and the diffusion time, do not appear to affect these modes. ${ }^{50}$ The scaling law for $\Gamma_{\text {leak }}$ was observed for decay rates as small as the numerical precision of our simulations allows $\left(\sim 10^{-14}\right)$. This suggests that if there exists another natural linewidth for localized modes, that does not originate in finite-size effects, it may be extremely small, possibly beyond the timescales accessible in an experiment.

\subsection{Intensity Decay}

A macroscopic quantity derived from atomic dipole is the Emitted Intensity $I_{\tilde{r}}(t)$ at observer point $\tilde{r}$ and time $t$. This quantity can be experimentally understood as the light intensity of the cloud after the incident laser is switch-off. In spherical coordinates, we can write $I_{\theta, \phi}(t)$ in the form ${ }^{31}$

$$
I_{\theta, \phi}(t) \propto\left|\sum_{j=1}^{N} e^{-i k f_{j}(\theta, \phi)} \beta_{j}(t)\right|^{2}
$$


where $f_{j}(\theta, \phi)=x_{j} \cos (\theta) \sin (\phi)+y_{j} \sin (\theta) \sin (\phi)+z_{j} \cos (\theta)$ and $N$ is the number of atoms. Using a plane wave laser in $\hat{z}$ direction creates an azimuthal symmetry that suggests integrating equation 3.4 over $\phi$ :

$$
I_{\theta}(t) \propto \int_{0}^{2 \pi} I_{\theta, \phi}(t) d \phi=\int_{0}^{2 \pi}\left|\sum_{j=1}^{N} e^{-i k f_{j}(\theta, \phi)} \beta_{j}(t)\right|^{2} d \phi
$$

The value of $\theta$ was fixed at $35^{\circ}$ and the $\phi$ angular integral become a discrete sum, dividing $\phi$ angular domain in $n_{\phi}=30$ steps. Initial dipole were considered the steadystate:

$$
\beta_{s}=\frac{i}{2} M^{-1} \Omega
$$

and its time evolution obeys $\beta(t)=e^{M t} \beta_{s}$. To reduce the number of operations, we use the relation $e^{A}=e^{Y X Y}=Y e^{X} Y^{-1}$, with $Y=\Psi$ e $X=\lambda$ :

$$
\beta(t)=\Psi e^{\lambda t} \Psi^{-1} \beta_{s}
$$

In equation 3.7 only the term $e^{\lambda t}$ is computed, providing a substantial speed up. However, a slightly performance hack is still available. Instead of multiplying 3 matrices and one matrix-vector multiplication as in equation 3.7, we defined a Time Evolution Matrix Operator $e^{M \delta t}=\Psi e^{\lambda \delta t} \Psi^{-1}$ (time difference is a constant $\delta t=\left|t_{n+1}-t_{n}\right|^{*}$ ) and compute $\beta$ iteratively

$$
\beta_{t+1}=e^{M \delta t} \beta_{t}
$$

Using the initial condition :

$$
\beta_{0}=\Psi e^{\lambda t_{0}} \Psi^{-1} \beta_{s}
$$

Anderson localization signature in past experiments has been deviations of exponential decay in transmission profiles ${ }^{6,22}$, we look for $I_{\theta}(t)$ to express this expectation. In Figure 11 we investigate how $I_{\theta}(t)$ changes with the detuning, and in two regimes: one below and other above the critical density. At resonance $(\Delta=0)$, both regimes presents exponential decays that are related to radiation trapping. ${ }^{21,46}$ For low density, at Figure 11a, $I_{\theta}(t)$ follows an exponential law. In contrast, for $\rho \gg \rho_{c}$ in Figure 11b, intensity rates presents a power law decay, but presents an anomaly at $\Delta=1$, where the decay is strongly suppressed. This anomaly is not found at low density where as can be seen in Figure 11a the $\Delta=1$ curve is practically indistinguishable from the other curves.

* smaller the difference, better the result 
(a)

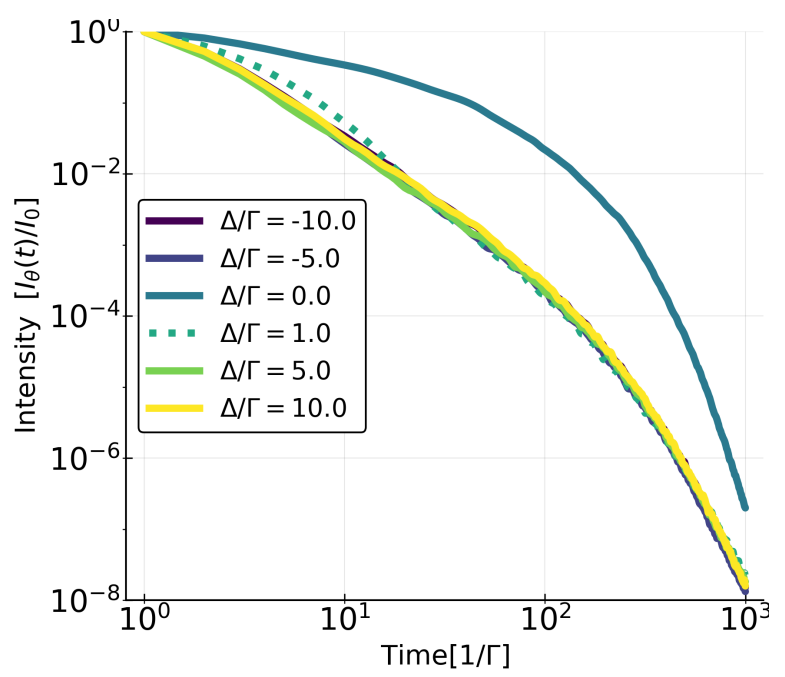

(b)

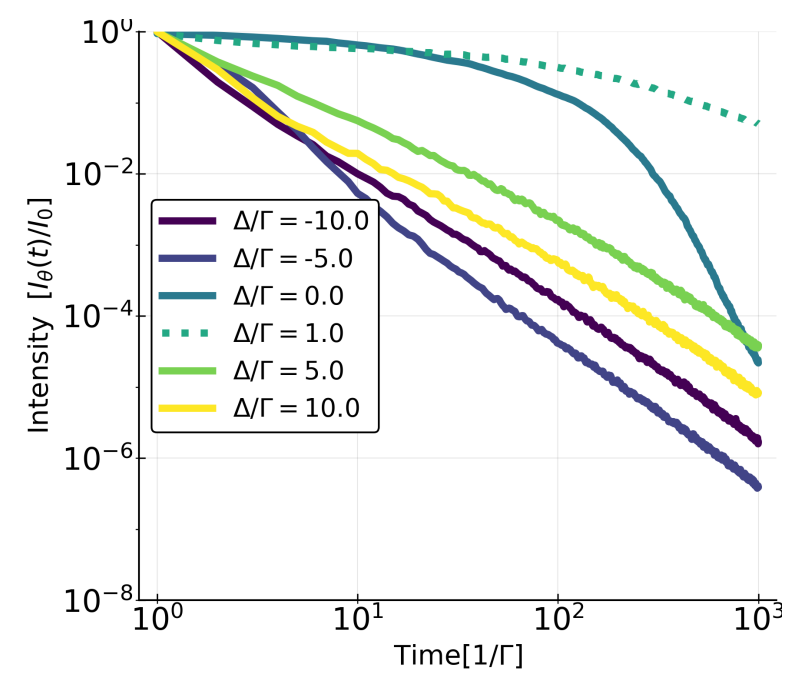

Figure 11 - Light decay for a system of $N=1500$ and averaged over 100 realizations. Source: By the author.

This anomaly is of particular interest since it occurs exactly when the strongest coupling of the laser with the localized modes is expected, see Figure 8. A systematic study of this behavior is needed (e.g., if these results hold for vectorial case), but, if true, this result suggests that future experiments may focus on the detuning to identify signatures of localization. 


\section{CONCLUSION}

In this work, we have studied the population of modes throughout the Anderson localization phase transition for 3D light scattering. The scenario where all modes localize, up to finite-size effects, clearly does not hold, as both spatially-extended subradiant and superradiant modes are present. Furthermore, even tuning the pump frequency close to the localized modes resonant energy, at most half of the incoming wave is observed to couple to localized modes, so the strong contribution from other modes to the transport of light cannot be excluded.

A natural question is whether the transport of light can really be put to a halt thanks to disorder, with the paradigmatic decay of the coherent transmission with the system length. ${ }^{6}$ For now, there is no such report in 3D configurations, but rather observations of an increase of the transport at higher densities, as compared to the diffusion prediction. ${ }^{51}$ The absence of analytical tools and the strong limitations of numerical simulations of 3D systems definitely represent an obstacle to understand the specificity of 3D light scattering. In this regard, the correlations between the lifetime of localized modes and the system size observed in our are a good illustration of the limits of simulating small systems. 



\section{REFERENCES}

1 ANDERSON, P. W. Absence of diffusion in certain random lattices. Physical Review, v. 109, n. 5, p. 1492-1505, 1958.

2 HU, H.; STRYBULEVYCH, A.; PAGE, J. H.; SKIPETROV, S. E.; TIGGELEN, B. A. van. Localization of ultrasound in a three-dimensional elastic network. Nature Physics, v. 4, n. 12, p. 945-948, 2008.

3 BILLY, J.; JOSSE, V.; ZUO, Z.; BERNARD, A.; HAMBRECHT, B.; LUGAN, P.; CLÉMENT, D.; SANCHEZ-PALENCIA, L.; BOUYER, P.; ASPECT, A. Direct observation of Anderson localization of matter waves in a controlled disorder. Nature, v. 453, n. 7197, p. 891-894, 2008.

4 KONDOV, S. S.; MCGEHEE, W. R.; ZIRBEL, J. J.; DEMARCO, B. Threedimensional Anderson localization of ultracold matter. Science, v. 334, n. 6052, p. 66-68, 2011.

5 SEMEGHINI, G.; LANDINI, M.; CASTILHO, P.; ROY, S.; SPAGNOLli, G.; TRENKWALDER, A.; FATTORI, M.; INGUSCIO, M.; MODUGNO, G. Measurement of the mobility edge for 3d Anderson localization. Nature Physics, v. 11, n. 7, p. 554-559, 2015.

6 BERRY, M. V.; KLEIN, S. Transparent mirrors: rays, waves and localization. European Journal of Physics, v. 18, n. 3, p. 222-228, 1997.

7 LAURENT, D.; LEGRAND, O.; SEBBAH, P.; VANNESTE, C.; MORTESSAGNE, F. Localized modes in a finite-size open disordered microwave cavity. Physical Review Letters, v. 99, n. 25, p. 253902, 2007.

8 SKIPETROV, S. E. Finite-size scaling analysis of localization transition for scalar waves in a three-dimensional ensemble of resonant point scatterers. Physical Review B, v. 94, n. 6, p. 064202, 2016.

9 GENACK, A. Z.; GARCIA, N. Observation of photon localization in a threedimensional disordered system. Physical Review Letters, v. 66, n. 16, p. 2064-2067, 1991.

10 WIERSMA, D. S.; BARTOLINI, P.; LAGENDIJK, A.; RIGHINI, R. Localization of light in a disordered medium. Nature, v. 390, n. 6661, p. 671-673, 1997.

11 SCHUURMANS, F. J. P.; MEGENS, M.; VANMAEKELBERGH, D.; LAGENDIJK, A. Light scattering near the localization transition in macroporous GaP networks. Physical Review Letters, v. 83, n. 11, p. 2183-2186, 1999.

12 SPERLing, T.; BüHRER, W.; AEGERTER, C. M.; MARET, G. Direct determination of the transition to localization of light in three dimensions. Nature Photonics, v. 7, n. 1, p. 48-52, 2012. 
13 STöRZER, M.; GROSS, P.; AEGERTER, C. M.; MARET, G. Observation of the critical regime near Anderson localization of light. Physical Review Letters, v. 96, n. 6 , p. $063904,2006$.

14 SCHEFFOLD, F.; LENKE, R.; TWEER, R.; MARET, G. Localization or classical diffusion of light? Nature, v. 398, p. 206-207, 1999. doi: 10.1038/18347.

15 SPERLING, T.; SCHERTEL, L.; ACKERMANN, M.; AUBRY, G. J.; AEGERTER, C. M.; MARET, G. Can 3d light localization be reached in 'white paint'? New Journal of Physics, v. 18, n. 1, p. 013-039, 2016.

16 SKIPETROV, S.; SOKOLOV, I. Absence of Anderson localization of light in a random ensemble of point scatterers. Physical Review Letters, v. 112, n. 2, p. 023905, 2014.

17 MÁximO, C. E.; PIOVElla, N.; COURTEIlle, P. W.; KAISER, R.; BACHELARD, R. Spatial and temporal localization of light in two dimensions. Physical Review A, v. 92, n. 6, p. 062702, 2015.

18 ABRAHAMS, E.; ANDERSON, P. W.; LICCIARDELLO, D. C.; RAMAKRISHNAN, T. V. Scaling theory of localization: absence of quantum diffusion in two dimensions. Physical Review Letters, v. 42, n. 10, p. 673-676, 1979.

19 DICKE, R. H. Coherence in spontaneous radiation processes. Physical Review, v. 93, n. 1, p. 99-110, 1954.

20 PAVOlini, D.; CRUBEllier, A.; PILlet, P.; CABARET, L.; LIBERMAN, S. Experimental evidence for subradiance. Physical Review Letters, v. 54, n. 17, p. 1917-1920, 1985.

21 GUERIN, W.; ARAÚJO, M. O.; KAISER, R. Subradiance in a large cloud of cold atoms. Physical Review Letters, v. 116, n. 8, p. 083601, 2016.

22 COTTIER, F.; KAISER, R.; BACHELARD, R. Role of disorder in super- and subradiance of cold atomic clouds. Physical Review A, v. 98, n. 1, p. 013622, 2018.

23 SKIPETROV, S.; SOKOLOV, I. Magnetic-field-driven localization of light in a cold-atom gas. Physical Review Letters, v. 114, n. 5, p. 053902, 2015.

24 BELLANDO, L.; GERO, A.; AKKERMANS, E.; KAISER, R. Cooperative effects and disorder: a scaling analysis of the spectrum of the effective atomic hamiltonian.

Physical Review A, v. 90, n. 6, p. 063822, 2014.

25 SKIPETROV, S. E.; SOKOLOV, I. M. Ioffe-Regel criterion for Anderson localization in the model of resonant point scatterers. Physical Review B, v. 98, n. 6, p. 064207, 2018.

26 SKIPETROV, S. Localization transition for light scattering by cold atoms in an external magnetic field. Physical Review Letters, v. 121, n. 9, p. 093601, 2018.

27 GARCIA, R.; ZOZULYA, A.; STICKNEY, J. MATLAB codes for teaching quantum physics: part 1. 2008. Available from: <https://arxiv.org/pdf/0704.1622.pdf>. Accessible at: 11 May 2019. 
28 KUTZ, J. N. Data-driven modeling and scientific computation: methods for complex systems and big data. New York: Oxford University Press, 2013. 657 p.

29 LEHMBERG, R. H. Radiation from anN-atom system. i. general formalism. Physical Review A, v. 2, n. 3, p. 883-888, 1970.

30 SVIDZINSKY, A. A.; CHANG, J.-T.; SCULLY, M. O. Cooperative spontaneous emission of $\mathrm{N}$ atoms: many-body eigenstates, the effect of virtual lamb shift processes, and analogy with radiation of $\mathrm{N}$ classical oscillators. Physical Review A, v. 81, n. 5, p. 053821, 2010.

31 ARAÚJO, M. O.; GUERIN, W.; KAISER, R. Decay dynamics in the coupled-dipole model. Journal of Modern Optics, v. 65, n. 11, p. 1345-1354, 2017.

32 BIELla, A.; BORGONOVI, F.; KAISER, R.; CELARDO, G. L. Subradiant hybrid states in the open 3d Anderson-Dicke model. EPL (Europhysics Letters), v. 103, n. 5, p. 57009, 2013.

33 CELARDO, G. L.; ANGELI, M.; KAISER, R. Localization of light in subradiant Dicke states: a mobility edge in the imaginary axis. 2017. Available from: <https://arxiv.org/abs/1702.04506>. Accessible at: 7 May 2019.

34 KE, Q.; KANADE, T. Robust 11 norm factorization in the presence of outliers and missing data by alternative convex programming. In: IEEE COMPUTER SOCIETY CONFERENCE ON COMPUTER VISION AND PATTERN RECOGNITION, 2005, San Diego. Proceedings... San Diego: IEEE, 2005. p. 739-746.

35 XIAO, Y.; ZENG, T.; YU, J.; NG, M. K. Restoration of images corrupted by mixed gaussian-impulse noise via 11-10 minimization. Pattern Recognition, v. 44, n. 8, p. 1708-1720, 2011.

36 KAISER, R.; MONTALDI, J. Peyresq Lectures on Nonlinear Phenomena. Singapore: World Scientific, 2000. 296 p.

37 KAISER, R. Quantum multiple scattering. Journal of Modern Optics, v. 56, n. 18-19, p. 2082-2088, 2009.

38 COTTIER, F.; CIPRIS, A.; BACHELARD, R.; KAISER, R. Intensity

fluctuations signature of 3D Anderson localization of light. 2019. Available from: $<$ https://arxiv.org/abs/1812.10313>. Accessible at: 3 Sept. 2019.

39 BELL, R. J. The dynamics of disordered lattices. Reports on Progress in Physics, v. 35, n. 3, p. 1315-1409, 1972.

40 PENDRY, J. B. Quasi-extended electron states in strongly disordered systems. Journal of Physics C: solid state physics, v. 20, n. 5, p. 733-742, 1987.

41 BerTOlOtTI, J.; GOTTARDO, S.; WIERSMA, D. S.; GHULINYAN, M.; PAVESI, L. Optical necklace states in Anderson localized 1d systems. Physical Review Letters, v. 94, n. 11, p. 113903, 2005.

42 EVERS, F.; MIRLIN, A. D. Anderson transitions. Reviews of Modern Physics, v. 80, n. 4 , p. $1355-1417,2008$. 
43 NAKAYAMA, K. Y. T. Fractal concepts in condensed matter physics. Berlin: Springer, 2010. 224 p. (Springer series in solid-state sciences).

44 RODRIGUEZ, A.; VASQUEZ, L. J.; SLEVIN, K.; RöMER, R. A. Multifractal finite-size scaling and universality at the Anderson transition. Physical Review B, v. 84, n. 13, p. 134209, 2011.

45 GUERIN, W.; KAISER, R. Population of collective modes in light scattering by many atoms. Physical Review A, v. 95, n. 5, p. 053865, 2017.

46 ARAÚJO, M. O.; KREŠIĆ, I.; KAISER, R.; GUERIN, W. Superradiance in a large and dilute cloud of cold atoms in the linear-optics regime. Physical Review Letters, v. 117, n. 7, p. 073002, 2016.

47 ROOF, S.; KEMP, K.; HAVEY, M.; SOKOLOV, I. Observation of single-photon superradiance and the cooperative lamb shift in an extended sample of cold atoms.

Physical Review Letters, v. 117, n. 7, p. 073003, 2016.

48 SCULLY, M. O.; FRY, E. S.; OOI, C. H. R.; WÓDKIEWICZ, K. Directed spontaneous emission from an extended ensemble of $\mathrm{N}$ atoms: timing is everything. Physical Review Letters, v. 96, n. 1, p. 010501, 2006.

49 PINHEIRO, F. A.; RUSEK, M.; ORLOWSKI, A.; TIGGELEN, B. A. van. Probing Anderson localization of light via decay rate statistics. Physical Review E, v. 69, n. 2, p. 026605, 2004.

50 AKKERMANS, E.; MONTAMBAUX, G. Mesoscopic physics of electrons and photons. Cambridge: Cambridge University Press, 2007. 608 p.

51 GUERIN, W.; ROUABAH, M.; KAISER, R. Light interacting with atomic ensembles: collective, cooperative and mesoscopic effects. Journal of Modern Optics, v. 64, n. 9, p. 895-907, 2016. 